\title{
Docking of B-cell epitope antigen to specific hepatitis B antibody
}

\author{
R RAJKANNAN $^{1}$ and E J PADMA MALAR ${ }^{2, *}$ \\ ${ }^{1}$ Department of Physical Chemistry, University of Madras, Guindy Campus, Chennai 600025 \\ ${ }^{2}$ National Centre for Ultrafast Processes, University of Madras, Taramani Campus, Chennai 600113 \\ e-mail: ejpmalar@yahoo.com
}

MS received 15 June 2007; accepted 7 July 2007

\begin{abstract}
The interaction of pres1 region of hepatitis B virus B-cell epitope antigen with specific hepatitis B neutralizing monoclonal antibody was examined by docking study. We modelled the 3D complex structure of B-cell epitope antigen residues CTTPAQGNSMFPSCCCTKPTDGNCY by homology modelling and docked it with the crystal structure of monoclonal antibody specific for the pres1 region of the hepatitis B virus. At the optimized docked conformation, the interactions between the amino acids of antigen and antibody were examined. It is found that the docked complex is stabilized by $59 \cdot 3 \mathrm{kcal} / \mathrm{mol}$. The stability of the docked antigen-antibody complex is due to hydrogen bonding and van der Waals interactions. The amino acids of the antigen and antibody responsible for the interaction were identified.
\end{abstract}

Keywords. Molecular docking; modelling antibody-antigen interaction; hepatitis B virus B-cell epitope antigen; hepatitis B neutralizing monoclonal antibody; homology modelling; antigen-antibody docking.

\section{Introduction}

Structural studies on interacting antibodies (Ab) and antigens (Ag) are highly beneficial to the understanding of principles that govern the interactions. Antibodies are remarkable molecules of immune system-related proteins called immunoglobulins. They differ not only in their physiological roles but also in their structures and serve as paradigms for macromolecular recognition. They have a binding site that is designed to accommodate ligands of variable structure (the binding site) and sites that bind to ligands of constant structure. ${ }^{1}$

A significant attribute of antibodies is their specificity in the binding of various types of antigens. This characteristic property is due to different kinds of interactions, such as charge-charge, dipole-dipole, hydrogen bonding, and van der Waals interactions between the antigen and residues present in the binding site. ${ }^{2,3}$ However, shape complementarity seems to play a major role in molecular recognition and can be used as central criterion for the antibody-antigen docking studies. ${ }^{4}$ Recently, computational techniques for docking potential ligands based on the shape of protein receptors have been developed, and the structural studies of antibody-antigen interac-

\footnotetext{
*For correspondence
}

tions implicate amino-acid side chains from complementarity determining regions (CDRs) of antibody in forming contacts with antigen.

Given the structure (or a model) of an antigen and the structure of an antibody raised against the antigen, can we predict the site of interaction between the two molecules? The solution to this problem, even if approximate, would help in designing experiments to precisely map the residues involved in the interface and could be instrumental both in designing peptides able to mimic the interacting surface of the antigen and in understanding where important regions of an antigen are located in its three-dimensional structure. Therefore, mapping the interaction surface between antibody and antigen that bind each other is of outstanding interest in molecular immunology.

Complementary to various experimental techniques, numerous computational methods exist for investigating the versatile binding characteristics of antibodies. A central position in this field is occupied by docking methods. Although their main area of application is within rational drug design, they are generally suited to elucidation of protein-ligand interactions and as such also in the investigation of antigen binding by antibodies. ${ }^{5}$

In this work, we have studied the interactions between pres1 region of hepatitis B virus B-cell epitope antigen with its specific hepatitis B neutralizing 
monoclonal antibody using molecular docking methods. B-cell epitope antigen is an immunogenic peptide comprising CTTPAQGNSMFPSCCCTKPTDGNCY amino acid sequence selected from the immunodominant B-cell epitope of hepatitis B surface antigen (HBsAg). Here, we illustrate the methodology of 3D model building for the antigen B-cell epitope and present the results of its docking with the specific hepatitis B neutralizing monoclonal antibody. Based on the interatomic distances, hydrogen bonding and energies the interactions between the amino acids of B-cell epitope antigen and its antibody were examined.

\section{Computational aspects}

\subsection{Homology modelling}

Homology modelling is the art of building a 3D protein structure for a new amino acid sequence (target) from the known complete 3D structure of at least one other reference protein (template). ${ }^{6}$ Proteins from different sources and sometimes diverse biological functions can have similar sequences and high sequence similarity is reflected by distinct structure similarity. Based on this fact, we build the 3D model structure for pres1 region of B-cell epitope peptide residues CTTPAQGNSMFPSCCCTKPTDGNCY by homology modelling from the available crystal structure of B-cell epitope in pres2 region of hepatitis $B$ virus (PDB code 1IUD) ${ }^{7}$ using SWISS-MODEL, an automated homology modelling server. ${ }^{8}$ The 3D crystal structure of monoclonal antibody specific for the pres1 region of the hepatitis B virus (PDB code $1 \mathrm{H} 3 \mathrm{P})^{9}$ was retrieved directly from protein data bank (figure 1).

Energy minimization calculations for the 3D model of B-cell epitope antigen and its specific antibody were performed using GROMOS 96 4B1 parameter set energy minimization package ${ }^{10}$ of SWISS PDB viewer. The 3D structure validation of the modeled B-cell epitope peptide antigen was carried out in SAVS info server. ${ }^{11}$ Active binding sites in pres1 region of hepatitis $\mathrm{B}$ neutralizing monoclonal antibody were identified using computed atlas of surface topography of proteins (CASTp) calculations. ${ }^{12}$

\subsection{Molecular docking}

Docking of B-cell epitope antigen to pres1 region Hepatitis B neutralizing monoclonal antibody was carried out using Hex 4.2 software. ${ }^{13}$ Docking simulations were carried out using the default full rotation mode, in which the antibody and antigen are effectively rotated about their own centroids, and the antigen is twisted about the intermolecular axis at each of a range of intermolecular distances. Thus the default behaviour is to perform a full six-dimensional search over the full rotational ranges. It was then accelerated by fast Fourier transform (FFT) docking algorithm which use a large cartesian grid to perform the Fourier transforms to accommodate all possible translations of the antigen about the stationary antibody. The default value in steric scan was used to improve the resolution in docking correlation. With suitable scaling factors, this docking score was interpreted as interaction energy.

\section{Results and discussion}

In this work we carried out docking studies among pres1 region of hepatitis B virus B-cell epitope antigen and its specific hepatitis B neutralizing monoclonal antibody. The purpose was to identify the most probable interaction zone of the antibody with antigen.

\subsection{Homology modelling of B-cell epitope antigen}

The crystal structure of B-cell epitope in pres2 region of hepatitis B virus (template) and the raw sequence

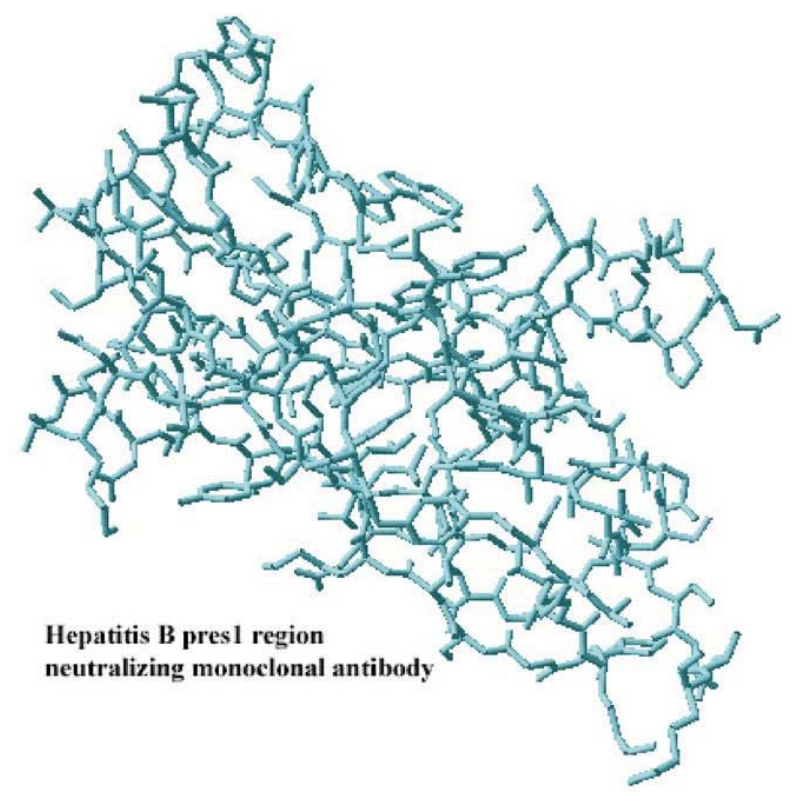

Figure 1. The 3D crystal structure of pres1 region hepatitis B neutralizing monoclonal antibody. 
of B-cell epitope antigen (target) was shown in figure 2 and the fitted model submitted to SWISS MODEL server for homology modelling was shown in figure 3. The 3D model of B-cell epitope antigen (figure 4) submitted to SAVS info structure validation server revealed that the overall quality factor of B-cell epitope antigen model was $100 \%$.

\subsection{Molecular docking}

The recognition and affinity of antibody towards antigen was interpreted from the interatomic distances and hydrogen bonding formed between the amino acid residues of docked antigen-antibody complex structure (figure 5). The interaction of B-cell epitope peptide amino acid sequence with antibody at interatomic distance less than $5 \AA$ were accounted in table 1 and shown in figure 6 .

The prominent binding pockets and cavities in hepatitis B antibody were identified using CASTp

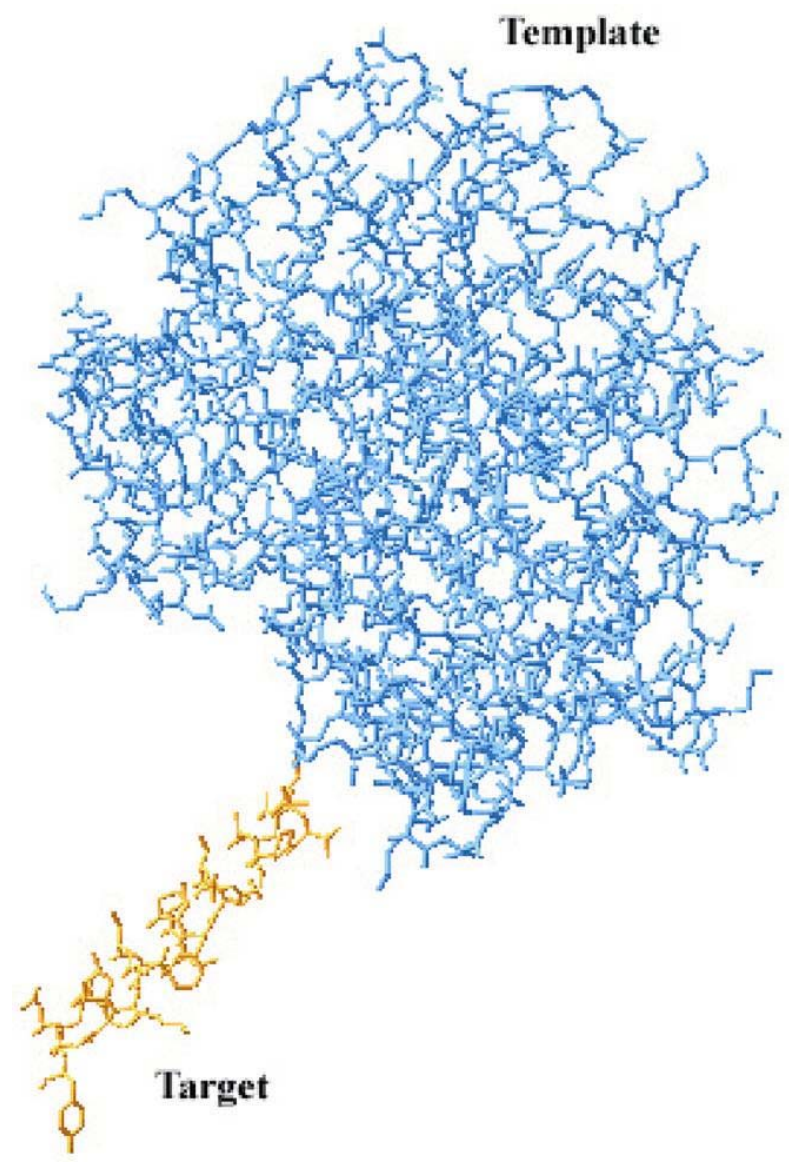

Figure 2. Structure of template and target raw sequence loaded in SWISS PDB viewer. calculations. CASTp is an online server used to predict the binding sites and active sites of proteins and DNAs and is often associated with structural pockets and cavities. CASTp calculations showed the surface accessible pockets as well as interior inaccessible

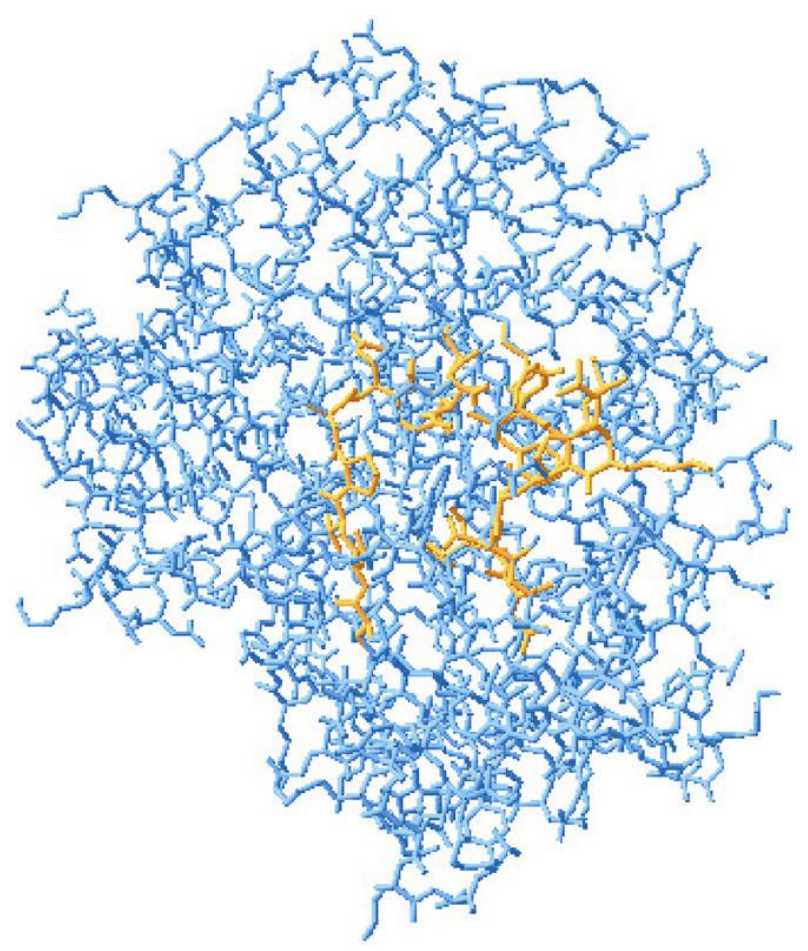

Figure 3. The fitted model submitted to SWISS MODEL server for homology modelling.

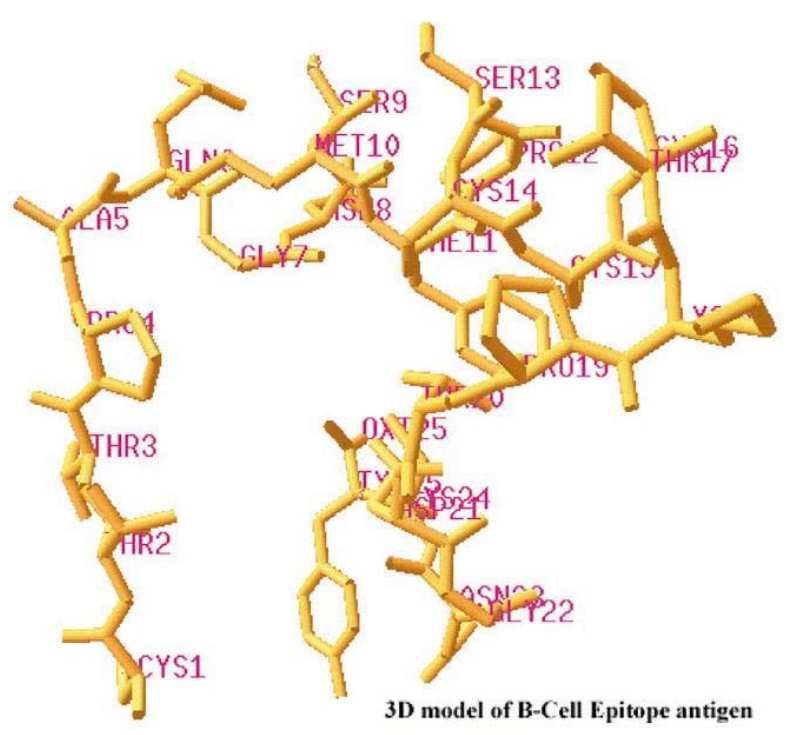

Figure 4. The 3D model of B-cell epitope peptide antigen. 


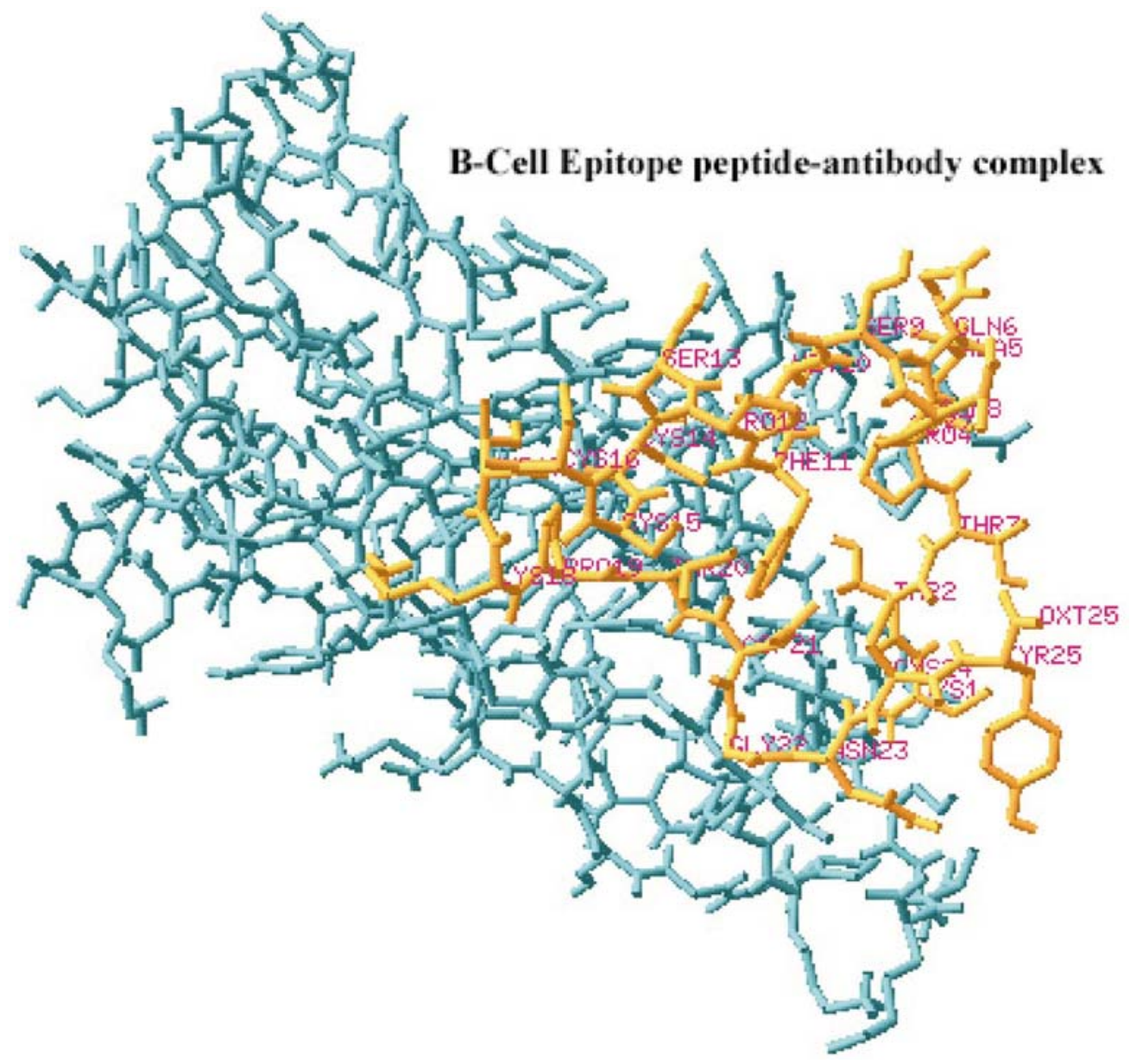

Figure 5. Docked B-cell epitope peptide antigen-antibody complex structure.

Table 1. Interaction of B-cell epitope peptide antigen amino acid sequence with antibody at interatomic distance less than $5 \AA$.

\begin{tabular}{llc}
\hline Antigen & Antibody & Distance $(\AA)^{*}$ \\
\hline Thr 2 CG2 & Thr 110 H & $3 \cdot 81$ \\
Pro 4 CA & Pro 41 O & $3 \cdot 40$ \\
Ala 5 O & Lys 43 H & $2 \cdot 23$ \\
Ser 13 O & Gln 105 H & $2 \cdot 40$ \\
Cys 14 O & Gln 105 CB & $3 \cdot 28$ \\
Lys 18 H & Glu 6 H & $4 \cdot 62$ \\
Thr 20 H & Gly 106 O & $4 \cdot 39$ \\
Thr 20 O & Thr 107 O & $4 \cdot 72$ \\
Asp 21 O & Met 89 CE & $3 \cdot 79$ \\
\hline
\end{tabular}

*CG2, Second $\gamma$ carbon (in the side chain); CA, $\alpha$ carbon; $\mathrm{CB}, \beta$ carbon; $\mathrm{CE}, \varepsilon$ carbon (in the side chain)

cavities of hepatitis B antibody. It showed the area and volume of each pocket and cavity, both in solvent accessible surface and molecular surface.
Three prominent active site pockets were identified involving both the heavy chain $(\mathrm{H})$ and light chain (L) amino acid residues of hepatitis B specific antibody. The major active site (figure 7 , green colour pocket) was contributed by amino acid residues of heavy chain Glu 1, Val 2, Gln 3, Leu 4, Val 5, Glu 6, Gln 39, Pro 41, Glu 42, Lys 43, Thr 87, Met 89, Tyr 91, Tyr 102, Trp 103, Gly 104, Gln 105, Leu 108, Thr 110, Tyr 145, Phe 146, Pro 147, Glu 148, Pro 149, Val 150, Thr 151, Val 152, Thr 153, Trp 154, Ser 156, Gly 157, Leu 159, Ser 160, Ser 161, Ser 162, Gly163, Val 164, His 165, Thr 166, Phe 167, Pro 168, Tyr 176, Leu 178 along with light chain amino acid residues Ser 9, Ser 10, Gln 38, Lys 39, Pro 40, Gly 41, Gln 42, Ser 43, Leu 83, Ala 84, Val 85, Tyr 87, Gly 100, Gly 101, Thr 102, Lys 103, Leu 104, Glu 105, Asn 138, Lys 142, Thr 164, Asp 165, Gln 166, Asp 167, Ser 168, Thr 172, Tyr 173 and Ser 174. This active site was buried inside the antibody, occupying 
an area of $1627.5 \AA^{2}$ and volume of $4334.3 \AA^{3}$. The side chains from Ala, Phe, Leu, Met, Pro, Val, Trp and Tyr account for the hydrophobic character of this pocket while the hydroxyl groups from Ser and Thr residues contribute to its hydrophilicity.

The second active site comprising both heavy and light chain amino acid residues occupying an area of $440 \cdot 2 \AA^{2}$ and volume of $440.4 \AA^{3}$ was located left to the prominent active site of hepatitis $\mathrm{B}$ antibody (figure 7 , orange colour pocket). The residues from light chain that surround the pocket are Tyr 32, Leu 33, Ala 34, Phe 36, Lys 45, Met 46, Tyr 49, Trp 50, Glu 55, Lys 89, Ser 91 and Tyr 96. The residues from heavy chain that surround the second active site are Tyr 32, Ala 33, Glu 50, Ser 94, Phe 96, Asn 97, Trp 98, Ala 101, Tyr 102 and Trp 103.

The third active site was located right to the prominent buried active site (see figure 7 , yellow colour pocket) occupying 291.6 $\AA^{2}$ area and $574.5 \AA^{3}$ volume. Heavy chain residues Pro 126, Gly 127, Ser 128, Asn 133, Met 135, Val 136, Thr 137, Leu 138, Thr 183, Lys 207, Ser 208, Phe 209 and light chain residues Thr 114, Val 115, Ser 116, Ile 117, Phe 118, Pro 119, Lys 207, Ser 208 and Phe 209 formed this small pocket.

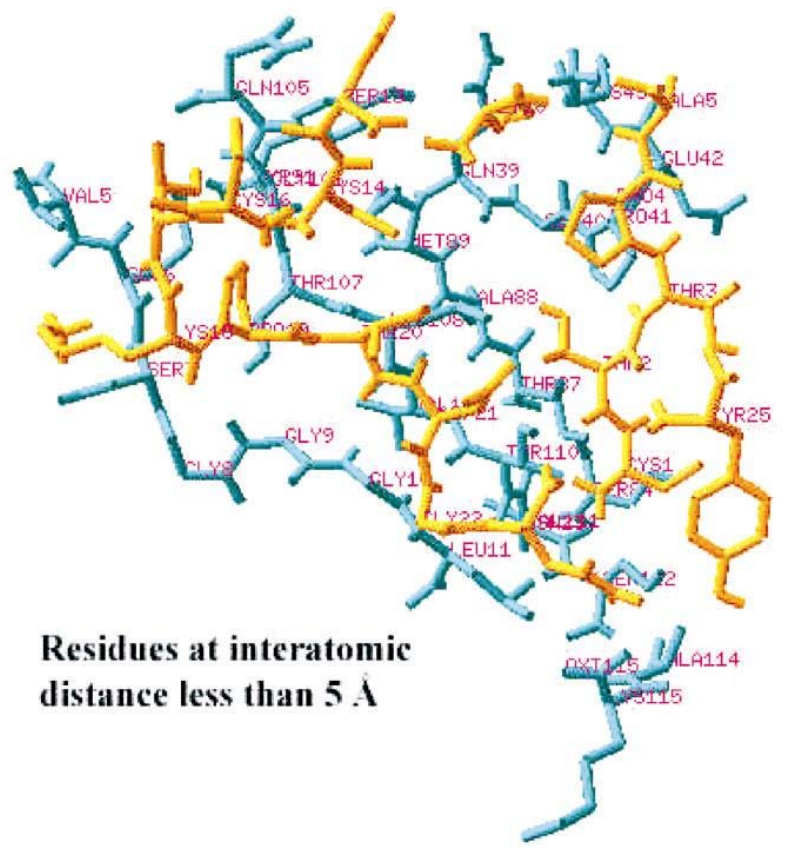

Figure 6. Interaction of B-cell epitope peptide amino acid sequence with antibody at interatomic distance less than $5 \AA$.
Apart from these active sites, another major active site in heavy chain was identified at the base of the major prominent active site pocket of the antibody (figure 7 , blue colour pocket) occupying $124 \cdot 7 \AA^{2}$ area and $163.3 \AA^{3}$ volume. The residues from the heavy chain that surround this pocket are Ser 7, Gly 8, Gly 9, Gly 10, Thr 107, Leu 108, Thr 110, Pro 147, Glu 148, Pro 149, Pro 201 and Ala 202.

The interaction of B-cell epitope peptide amino acid sequence with antibody at interatomic distance less than $5 \AA$ showed that the interactions between the antigen and antibody had occurred only in the active site pockets of hepatitis B antibody. The docking study reveals that van der Waals forces play an important role in stabilizing the antigen-antibody complex. The polar side chain group of threonine initiated the interaction between $\gamma 2$ carbon of Thr 2 in antigen and hydrogen of Thr 110 of the antibody. Threonine has a short group ended with a hydroxyl group which acts as hydrogen donor in enzymes and proteins. The N-end of proline residue forms an unusual imine ring along with the hydrophobic side chain forming non-covalent interaction between $\alpha$ carbon of Pro 4 in antigen and oxygen atom of Pro 41 of the antibody (table 1).

The high affinity of antigen towards antibody was favoured by four hydrogen bonds, formed by Cys 1 , Ala 5, ser 13 and Thr 20 of antigen residues with the antibody (figure 8). It is found that the following four hydrogen bonds involving (a) Donor hydrogen from Thr 87 of antibody with acceptor oxygen of Cys 1 in antigen: O-H...OC; (b) Donor hydrogen from Ala 5 of antigen with acceptor nitrogen of Lys 43 in antibody: $\mathrm{N}-\mathrm{H} \cdots \mathrm{N}^{+} \mathrm{H}_{3}$; (c) Donor hydrogen from Gln 105 of antibody with acceptor oxygen of Ser 13 in antigen: $\mathrm{NH}_{2} \cdots \mathrm{O}-\mathrm{H}$; (d) Donor hydrogen from Thr 20 of antigen with acceptor oxygen of Thr 107 in antibody: $\mathrm{NH} \cdots \mathrm{O}-\mathrm{H}$ are in the major active site region of hepatits $\mathrm{B}$ antibody. The hydrogen bond lengths $\mathrm{H} \cdots \mathrm{N}$ or $\mathrm{H} \cdots \mathrm{O}$ in these cases are found to be in the range 2.2 to $2.5 \AA$. The van der Waals interaction and hydrogen bonding formed by the reactive amino acid residues of antigenic peptide with the amino acids in the energy pockets of antibody lead to binding of antibody to antigen.

The docking study reveals that the antigen-antibody complex is stabilized by $59.3 \mathrm{kcal} / \mathrm{mol}$ as compared to the energies of free antigen and antibody. This energy lowering in the antibody-antigen complex is mainly due to the stabilizing hydrogen bonding and van der Waals interactions as seen earlier. 


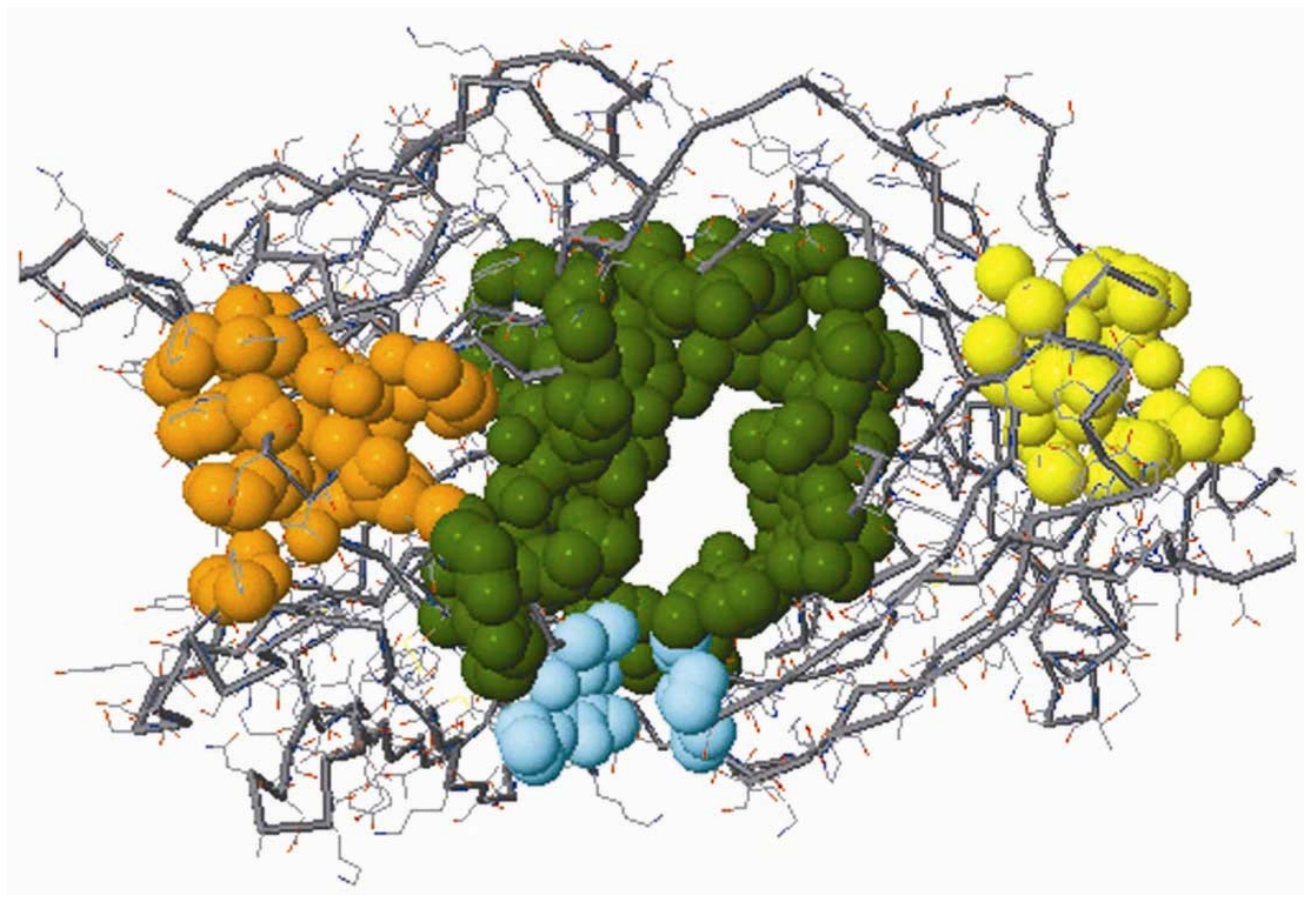

Figure 7. Active binding sites in hepatitis B antibody.



Figure 8. Hydrogen bonds formed between antigenantibody complex.

\section{Conclusion}

Docking of B-cell epitope peptide antigen to pres1 region hepatitis $B$ neutralizing monoclonal antibody facilitated the identification of amino acid sequences responsible for the interactions between these proteins. The basis of recognition and affinity was mainly due to formation of hydrogen bonding between antigen and antibody and also by the van der Waals forces.

\section{References}

1. Yuriev E and Ramsland P A 2001 J. Mol. Recog. 14172

2. Valjakka J, Hemminki A, Teerinen T, Takkinen K and Rouvinen J 2000 Acta Crystallogr. D Biol. Crystallogr. 56218

3. Valjakka J, Hemminki A, Niemi S, Soderlund H, Takkinen K and Rouvinen J 2002 J. Biol. Chem. 277 44021

4. Feng J and Li Y 2005 Immunol. Lett. 98311

5. Sotriffer C A, Flader W, Winger R H, Rode B M, Liedl K R and Varga J M 2000 Methods 20280

6. Bajorath J and Stenkamp B 1993 Protein Sci. 21798

7. Saul F A and Vulliez-le Normand B 1997 Proteins 271

8. http://www.expasy.org.spdbv/

9. Pizarro J C and Vulliez-le Normand B 2001 FEBS Lett. 509463

10. http://igc.ethz.ch/gromos/

11. http://nihserver.mbi.ucla.edu/savs/

12. Dundas J, Ouyang Z, Tseng J, Binkowski A, Turpaz, Y and Liang J 2006 Nucl. Acids Res. 34 W116

13. http://www.biochem.abdn.ac.uk/hex/ 